\title{
Traumatic Brain Injury: A Perspective on the Silent Epidemic
}

Ali Alkhaibary ${ }^{1,2,3}$, Abdulaziz Alshalawi ${ }^{4}$, Raad M. M. Althaqafi ${ }^{4}$, Abdullah A. Alghuraybi ${ }^{4}$, Ali Basalamah ${ }^{5}$, Ahmed M. Shammaa ${ }^{6}$, Ali A. Altalhy ${ }^{7}$, Tamer M. Abdelrahman ${ }^{4}$

1. Division of Neurosurgery, Department of Surgery, King Abdulaziz Medical City, Ministry of National Guard - Health Affairs, Riyadh, SAU 2. Neurosurgery, King Abdullah International Medical Research Center, Riyadh, SAU 3. College of Medicine, King Saud bin Abdulaziz University for Health Sciences, Riyadh, SAU 4. Department of Surgery, College of Medicine, Taif University, Taif, SAU 5. Department of Neurosurgery, King Saud University Medical City/King Khalid University Hospital, Riyadh, SAU 6. Department of Surgery, College of Medicine, Medical University of Warsaw, Warsaw, POL 7. Department of Neurosurgery, King Faisal Medical Complex, Taif, SAU

Corresponding author: Ali Alkhaibary, alkhaibarya@hotmail.com

\begin{abstract}
Background: Epidemiological data regarding the causes, patterns, severity, and outcomes of traumatic brain injury (TBI) are essential to plan for preventive strategies addressing this public health epidemic. The main aim of this study is to explore the patterns and causes of traumatic brain injury at two trauma centers.

Methods: A retrospective cohort study was conducted using a pre-tested validated data collection sheet. Data were collected from the medical records and electronic database of patients who presented to the emergency department with head trauma. Variables including the mechanisms, patterns of the injury, accompanying injuries, level of consciousness, and hospitalization duration were investigated for any possible association.
\end{abstract}

Results: A total of 269 patients (78\% males, 22\% females) who satisfied our study criteria were included in the final analysis. Motor vehicle collisions were the most common reason for traumatic brain injury (57.6\%) followed by falls (28.3\%). There was a statistically significant association observed between type of hemorrhage and Glasgow coma scale at initial presentation $(\mathrm{P}<0.05)$.

Conclusion: The most common cause of traumatic brain injury is motor vehicle collisions, followed by falls. The public should be made aware of the importance of using safety and precautionary measures to minimize the impact of traumatic brain injuries. Educational programs for neurotrauma prevention can be developed and utilized as a blueprint for local hospitals and officials in the country.

Review began 05/08/2021 Review ended 05/15/2021 Published 05/29/2021

\section{๑) Copyright 2021}

Alkhaibary et al. This is an open access article distributed under the terms of the Creative Commons Attribution License CC-BY 4.0., which permits unrestricted use, distribution, and reproduction in any medium, provided the original author and source are credited.

Categories: Neurosurgery

Keywords: collision, coma, head, injury, traffic

\section{Introduction}

Traumatic brain injury (TBI) is a silent public health epidemic and a major cause of disability, morbidity, and mortality worldwide [1]. TBI is characterized by a breakdown in the normal function of the brain caused by collision, blow, and jolt to the head-neck-spinal cord, possibly leading to temporary or permanent impairment [2]. Global epidemiological data show that approximately 69 million people suffer from TBI yearly. The highest incidence is reported in high-income countries compared to low-middle income countries [3].

Road traffic accidents are the leading cause of TBI. Higher rates of TBI in the United States are observed among older adults $(2,232$ per 100,000), followed by young children $(1,591$ per 100,000$)$ [4]. Although there is scarcity in the data regarding TBI from the Kingdom of Saudi Arabia, the reported incidence is 116 per 100,000 [5]. In Saudi Arabia, approximately 74\% of the cases of hemiplegia, paraplegia, and quadriplegia are due to motor vehicle collision (MVC) [6].

Due to the paucity of studies concerning TBI, the present study aimed to review the demographic information, mechanism of injury, severity of TBI, radiological findings on brain CT scan, length of stay (LOS), and the clinical/practical factors in traumatic brain injuries.

This manuscript was presented orally at the Annual Meeting of the Saudi Association of Neurological Surgery on $1 / 3 / 2020$.

\section{Materials And Methods}

\section{Study setting}


A retrospective cohort study was conducted using data collected from the medical records and electronic database of patients who presented to the emergency department with head trauma from April 2016 to April 2019. The study was conducted at two medical institutions, King Faisal Medical Complex and King Abdulaziz Specialized Hospital. These institutions provide medical care and advanced trauma care services to patients mainly from the Western region of Saudi Arabia. Data were collected and entered into a pre-designed and validated data collection sheet.

\section{Patient eligibility}

All patients who presented to the emergency department with traumatic head injuries during the study period were included. Initially, the records of 418 patients admitted to these two hospitals due to TBI were assessed. Patients with incomplete records and unsalvageable data were excluded from the analysis (35\%). These included missing charts, unrecognizable radiological records, and incomplete documentation of posttraumatic symptomatology.

\section{Data collection}

The examined variables included socio-demographic details, mechanism of injury, accompanying injuries, Glasgow coma scale (GCS) on admission, radiological findings on brain CT, length of stay (LOS), and symptomatology. The severity of TBI was assessed using the GCS. TBI was classified into mild (GCS = 15-13), moderate $(\mathrm{GCS}=12-9)$, and severe $(\mathrm{GCS}=8-3)$. The association between the mechanism of injury and gender was analyzed.

\section{Statistical analysis}

Data were entered into Microsoft Excel by an investigator. Statistical analysis was performed using SPSS version 23 (IBM Corp., Armonk, NY, USA) by an independent biostatistician. Categorical variables were summarized as proportions and frequencies. Any possible association of the variables was analyzed using Pearson's Chi-square test. Continuous variables were expressed as mean and standard deviation. A P-value of $\leqslant 0.05$ was considered statistically significant.

\section{Ethical considerations}

The ethical committees of the Research and Studies Department, Directorate of Health Affairs approved the current study. Prior to commencement of the study, permission was obtained from King Faisal Medical Complex and King Abdulaziz Specialized Hospital. Patient-related data were preserved and kept confidential. The identifying personal details were not included in the analysis. The assigned protocol number is HAP-02-T-067.

\section{Results}

\section{Baseline characteristics}

After scrutinizing the data, a total of 269 patients were included in the final analysis. The analysis included $22 \%$ females and $78 \%$ males who had some degree of TBI. The age distribution of the patients is illustrated in Figure 1. 


\section{Cureus}

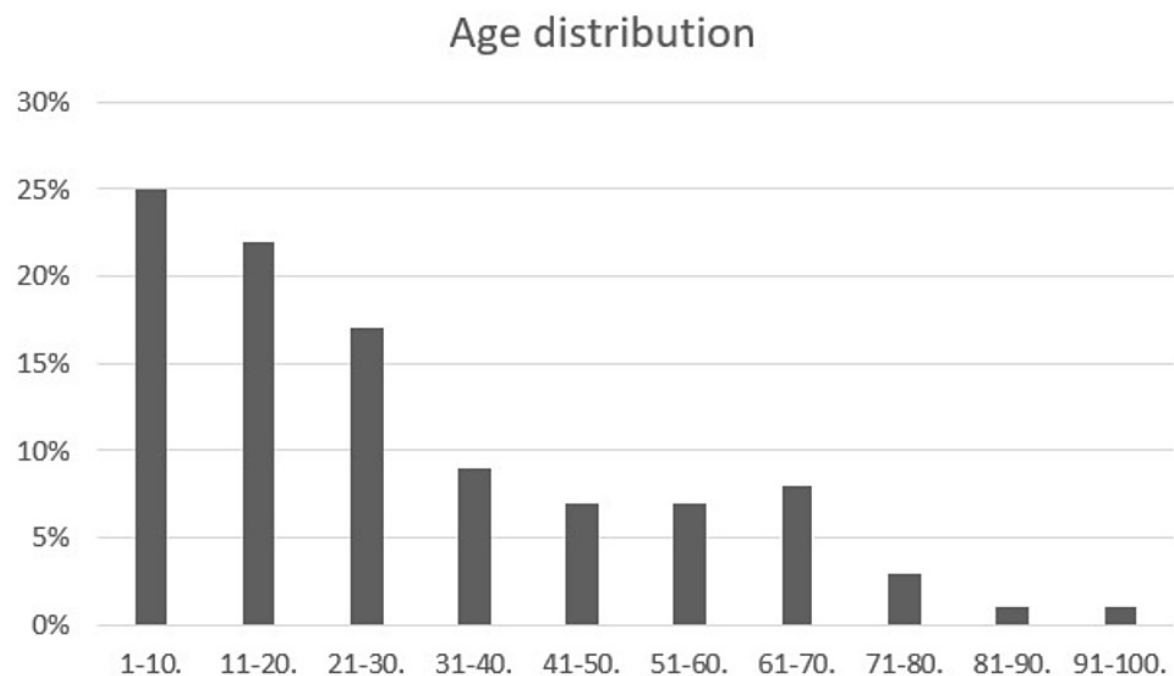

FIGURE 1: Age distribution of patients with traumatic brain injury presenting to the emergency department.

\section{Co-existing injury vs. type of hemorrhage}

When the co-existing injuries were assessed, it was found that $17.9 \%(n=10)$ of the patients with intra-axial hemorrhage had head and face injuries. Spinal cord injuries were noted in $5.7 \%(\mathrm{n}=5)$ of the patients with extra-axial hemorrhage. Table 1 outlines the type of hemorrhage and co-existing injuries.

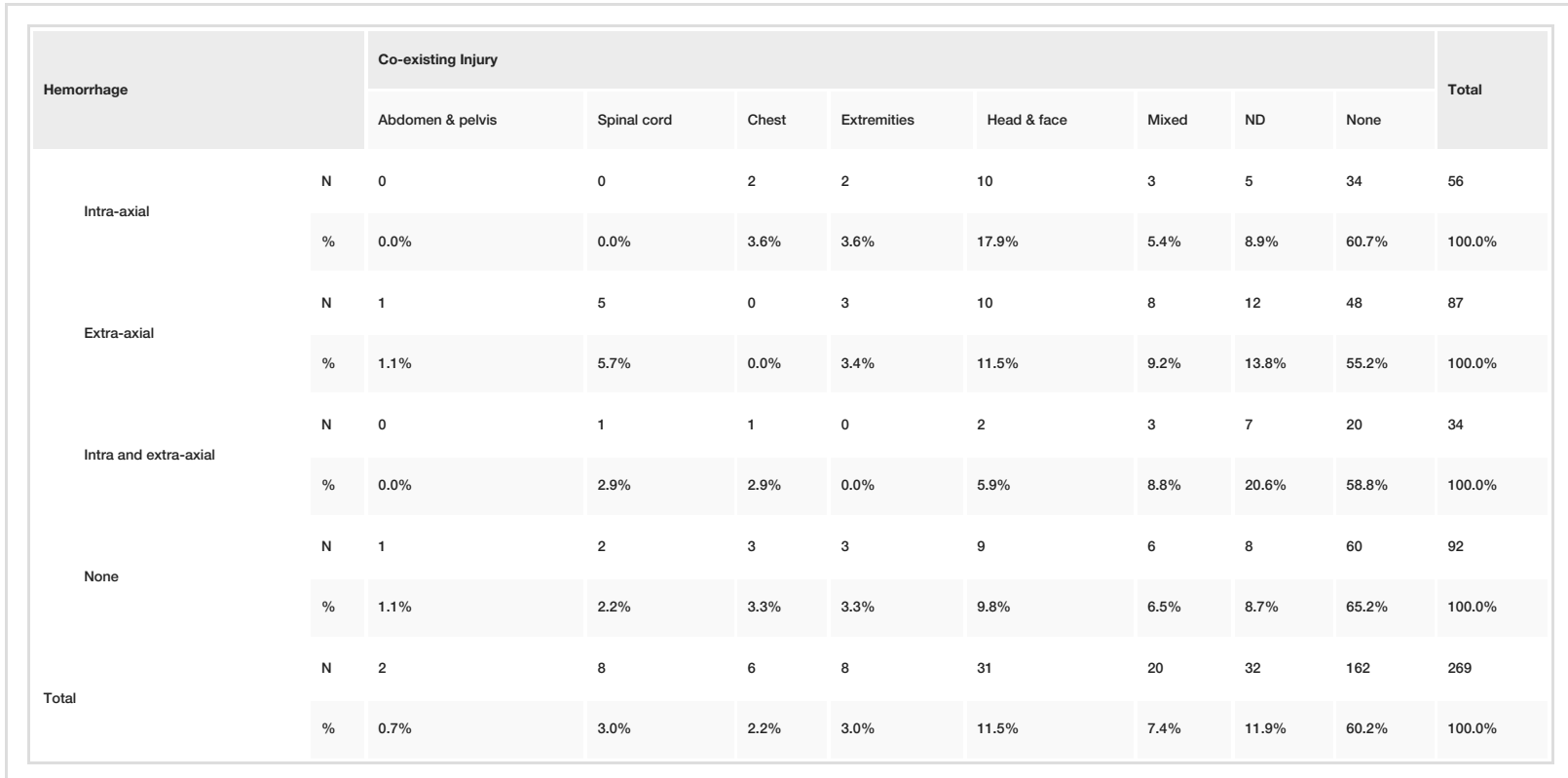

TABLE 1: Characteristics of the type of hemorrhage and the co-existing injuries.

ND: Not defined.

\section{Mechanism of injury vs. gender}

TBI was more common in males than females. Motor vehicle accidents in males accounted for $45.72 \%$ ( $n=$ 123) of trauma patients presenting to the emergency department. Table 2 outlines the mechanism of injury according to the gender of the patient. 


\section{Cureus}

\begin{tabular}{|c|c|c|c|c|c|c|c|c|c|}
\hline \multirow[b]{2}{*}{ Variable } & & \multicolumn{7}{|c|}{ Mechanism of Injury } & \multirow[b]{2}{*}{ Tota } \\
\hline & & Pedestrian & MVC & Motorcycle & Bike & Falls & Struck by objects & Assault & \\
\hline \multicolumn{10}{|l|}{ Gender } \\
\hline & Male & 5 & 123 & 5 & 3 & 57 & 1 & 16 & 210 \\
\hline & Female & 1 & 32 & 4 & 0 & 19 & 2 & 1 & 59 \\
\hline Total & & 6 & 155 & 9 & 3 & 76 & 3 & 17 & 269 \\
\hline
\end{tabular}

TABLE 2: Patterns of the mechanism of injury according to the gender of the patient.

MVC: Motor vehicle collision.

\section{Type of hemorrhage vs. Glasgow coma scale}

According to the GCS at initial presentation to the emergency department, $15.2 \%$ of the patients had severe TBI, $14.1 \%$ had moderate TBI, and $70.6 \%$ had mild TBI. When the type of hemorrhage was assessed, it was found that $19.5 \%$ of the patients with intra-axial hemorrhage had mild TBI. On the other hand, $28.9 \%$ of the patients with moderated TBI had extra-axial hemorrhage. The association between the type of hemorrhage and GCS was statistically significant $(\mathrm{P}=.001)$. Table 3 outlines the association between the type of hemorrhage and the severity of GCS.

\begin{tabular}{|c|c|c|c|c|c|c|c|}
\hline \multirow{2}{*}{ Glassow coma scale } & & \multicolumn{4}{|l|}{ Hemorrhage } & \multirow{2}{*}{ Total } & \multirow{2}{*}{ P-value } \\
\hline & & Intra-axial & Extra-axial & Intra and extra-axial & None & & \\
\hline \multirow{2}{*}{ Mild (15-13) } & $\mathrm{N}$ & 37 & 64 & 15 & 74 & 190 & \multirow{8}{*}{0.001} \\
\hline & $\%$ & $19.5 \%$ & $33.7 \%$ & $7.9 \%$ & $38.9 \%$ & $100.0 \%$ & \\
\hline \multirow{2}{*}{ Moderate (12-9) } & $\mathrm{N}$ & 10 & 11 & 6 & 11 & 38 & \\
\hline & $\%$ & $26.3 \%$ & $28.9 \%$ & $15.8 \%$ & $28.9 \%$ & $100.0 \%$ & \\
\hline \multirow{2}{*}{ Severe $(\leq 8)$} & $\mathrm{N}$ & 9 & 12 & 13 & 7 & 41 & \\
\hline & $\%$ & $22.0 \%$ & $29.3 \%$ & $31.7 \%$ & $17.1 \%$ & $100.0 \%$ & \\
\hline \multirow{2}{*}{ Total } & $\mathrm{N}$ & 56 & 87 & ${ }^{34}$ & 92 & 269 & \\
\hline & $\%$ & $20.8 \%$ & $32.3 \%$ & $12.6 \%$ & $34.2 \%$ & $100.0 \%$ & \\
\hline
\end{tabular}

TABLE 3: Association between the type of hemorrhage and Glasgow coma scale.

\section{Mechanism of injury vs. fracture type}

It was observed that a fracture of the vertebrae was more prevalent in MVCs. Fractures involving the base of the skull were noted in those who had a fall. Fractures involving the vault of the skull were more prevalent in assault. Table 4 outlines the type of fracture and the reason for injury. 


\section{Cureus}

\begin{tabular}{|c|c|c|c|c|c|c|c|c|}
\hline \multirow[b]{2}{*}{ Variable } & & \multicolumn{7}{|c|}{ Mechanism of injury } \\
\hline & & Pedestrian & Mvc & Motorcycle & Bike & Falls & Struck by objects & Assault \\
\hline \multicolumn{9}{|l|}{ Fracture } \\
\hline & Facial & 0 & 6 & 2 & 0 & 2 & 2 & 2 \\
\hline & Skull vault & 0 & 20 & 1 & 0 & 9 & 0 & 6 \\
\hline & Skul base & 1 & 2 & 1 & 0 & 5 & 0 & 0 \\
\hline & Vertebra & 1 & 6 & 0 & 0 & 0 & 0 & 0 \\
\hline & Mixed & 0 & 6 & 0 & 0 & 7 & 0 & 0 \\
\hline & None & 3 & 96 & 5 & 3 & 46 & 1 & 6 \\
\hline & ND & 1 & 19 & 0 & 0 & 7 & 0 & 3 \\
\hline & Total & 6 & 155 & 9 & 3 & 76 & 3 & 17 \\
\hline
\end{tabular}

TABLE 4: Characteristics of the type of fracture and the mechanism of injury.

MVC: Motor vehicle collision.

ND: Not defined.

\section{Type of fracture vs. hemorrhage}

Fracture of the vault of the skull was noted in $17.2 \%(n=15)$ of the patients with extra-axial hemorrhage. It was observed that a fracture of the vertebrae was more prevalent in patients with simultaneous intra- and extra-axial hemorrhage. Table 5 outlines the patterns of the type of hemorrhage and the type of fracture.

\begin{tabular}{|c|c|c|c|c|c|c|c|c|c|}
\hline \multirow{2}{*}{ Hemorrhage } & & \multicolumn{7}{|l|}{ Type of fracture } & \multirow{2}{*}{ Total } \\
\hline & & Vault of the skull & Base of the skull & Vertebra & Complex & Facial bones & ND & No fracture & \\
\hline \multirow{3}{*}{ Intra-axial } & $\mathrm{N}$ & 7 & 1 & 1 & 2 & 3 & 6 & 36 & 56 \\
\hline & $\%$ & $12.5 \%$ & $1.8 \%$ & $1.8 \%$ & $3.6 \%$ & $5.4 \%$ & $10.7 \%$ & $64.3 \%$ & $100.0 \%$ \\
\hline & $\mathrm{N}$ & 15 & 6 & 2 & 7 & 4 & 14 & 39 & 87 \\
\hline & $\%$ & $17.2 \%$ & $6.9 \%$ & $2.3 \%$ & $8.0 \%$ & $4.6 \%$ & $16.1 \%$ & $44.8 \%$ & $100.0 \%$ \\
\hline \multicolumn{10}{|c|}{ Intra and extra-axial } \\
\hline & $\%$ & $20.6 \%$ & $2.9 \%$ & $8.8 \%$ & $5.9 \%$ & $5.9 \%$ & $8.8 \%$ & 47.1\% & $100.0 \%$ \\
\hline \multicolumn{10}{|l|}{ None } \\
\hline & $\%$ & $7.6 \%$ & $1.1 \%$ & $1.1 \%$ & $2.2 \%$ & $5.4 \%$ & $7.6 \%$ & $75.0 \%$ & $100.0 \%$ \\
\hline \multirow{2}{*}{ Total } & $\mathrm{N}$ & 36 & 9 & 7 & 13 & 14 & 30 & 160 & 269 \\
\hline & $\%$ & $13.4 \%$ & $3.3 \%$ & $2.6 \%$ & $4.8 \%$ & $5.2 \%$ & $11.2 \%$ & $59.5 \%$ & $100.0 \%$ \\
\hline
\end{tabular}

TABLE 5: Patterns of the type of hemorrhage and type of fracture.

\section{Length of stay vs. type of hemorrhage}

The analysis of CT scan findings showed that $20.8 \%$ of the patients had intra-axial hemorrhage, $32.3 \% \%$ $(n=87)$ had extra-axial hemorrhage, and $12.6 \%$ had simultaneous intra- and extra-axial hemorrhage. When the association between the type of hemorrhage and the length of stay was assessed, it was found that most 
patients $(n=196 ; 66.2 \%)$ were hospitalized for less than one week $(P=0.007)$. Table 6 outlines the association between type of hemorrhage and the length of stay during hospitalization.

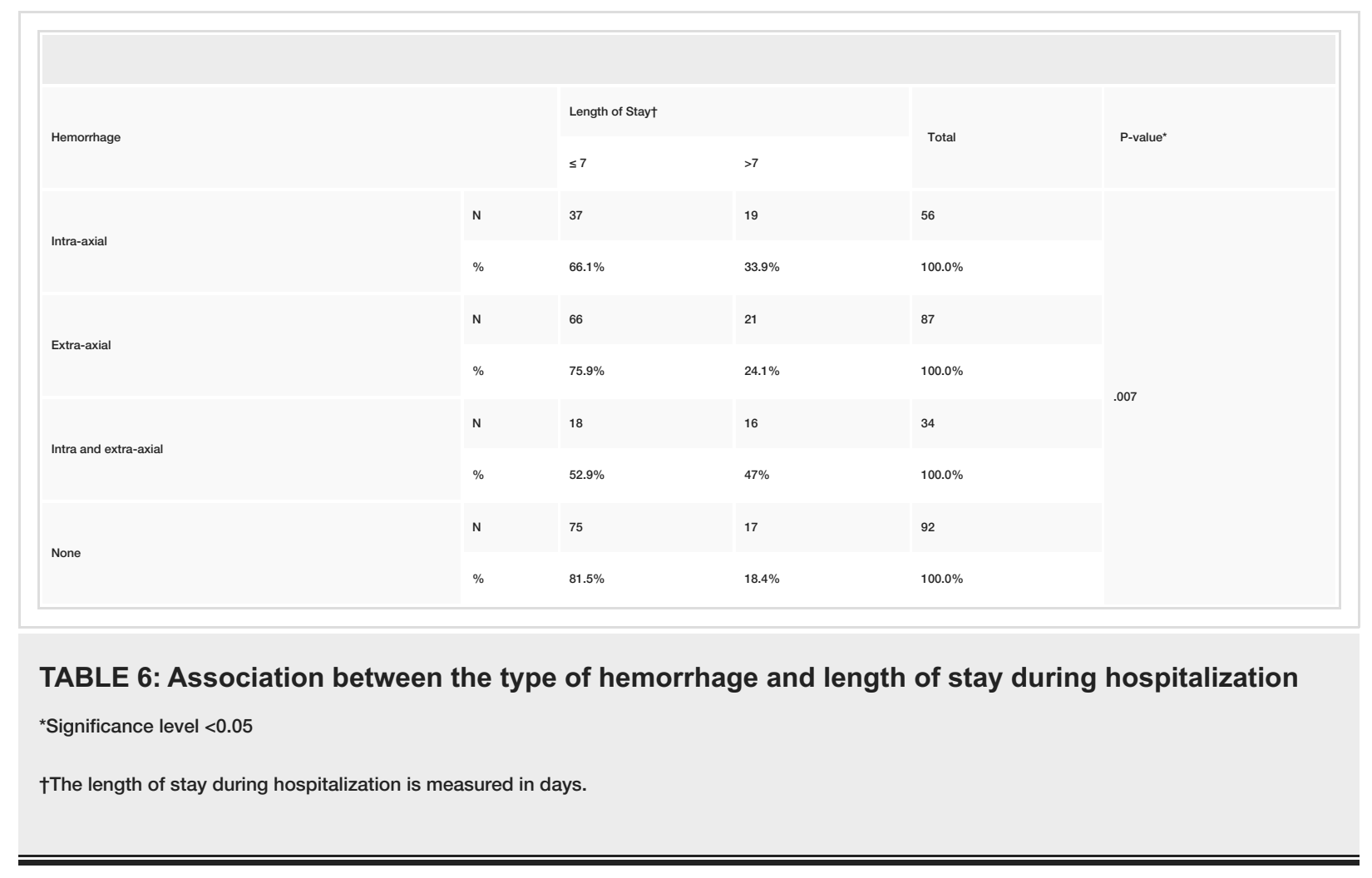

\section{Discussion}

Reports regarding TBI vary widely between and within countries due to the lack of data regarding the types, causes, and outcomes of TBI. The present study is a hospital-based, retrospective cohort analysis conducted at two medical institutions in Saudi Arabia. The findings of the study demonstrated that motor vehicle collisions (MVCs) were the leading cause of TBI. This concurs with other studies conducted in Middle Eastern countries, i.e. the United Arab Emirates and Qatar [7-8].

In contrast, a study in the United States reported that falls from heights and assault were the leading causes of TBI. However, MVCs remained the leading cause of death, followed by falls from heights [9]. In the present study, most patients with TBI were males and the leading cause of TBI in males was MVCs, although there was no significant association. This could be due to the fact that females were not allowed to drive during the major period of our study compared to other countries [7-9].

\section{Associated injuries}

Brain injuries resulting from MVCs, assault, or falls from heights frequently cause concurrent injuries to other body regions, including the spine, vertebrae, and extremities, the leading cause of death and disability [10]. The findings of the present study showed that head and spine injuries were commonly noted in MVCs and physical assaults. Studies show that one-third to one-half of the patients with severe extra-cranial injuries with associated brain injury have high mortality rates [11-13]. The reason for this could be explained on the basis that patients with injury at the extra-cranial sites could have massive hemorrhage, which could result in coagulopathy or decreased cerebral blood flow, causing secondary brain damage [14-15].

\section{Severity of brain injury}

The GCS is commonly recorded soon after arrival or within less than one hour after arrival to the hospital [16]. In our study, $15.2 \%$ of patients had severe brain injury whereas $14.1 \%$ had moderate brain injury. It was found that the severe brain injuries were noted in patients who had fractures of the vault of the skull, base of the skull, and vertebrae compared to other types of fracture. This finding was in accordance with the findings of Meng and Shi [17].

In another study conducted in the United States, it was reported that $14.5 \%$ of the patients who had severe brain injury had achieved good outcome at six months [18]. Studies also reported that $100 \%$ of mortality was observed in cases with fixed and dilated pupils who had severe brain injury [19-20]. 


\section{Hospitalization}

It is reported that patients with intracerebral hemorrhage (ICH) due to trauma suffer from long and uncertain hospitalization [21]. Therefore, it is important to take this into consideration to predict the outcomes and help healthcare providers take precautionary measures when managing patients with TBI. Studies report that various injury preventive programs were effective in reducing mortality and disability in patients who had road traffic accidents [22-23].

\section{Age at risk}

In the present study, the majority of victims with TBI (47\%) were 20 years of age and younger. This emphasizes the issue within the affected demographic. Similarly, El-Matbouly et al. conducted a retrospective study on TBI across age groups in Qatar. Their findings showed that the majority of victims were young adults (34\%) and middle-aged (21\%). This concurs with the findings of the present study. Therefore, young adolescents should be properly made aware of traumatic brain injury and how it can be a silent epidemic [24].

\section{Concurrent maxillofacial injury}

Aldwsari et al. prospectively explored maxillofacial traumas in patients who sustained road traffic accidents from 2013 to 2018. The authors highlighted the important association of maxillofacial traumas with traumatic brain injuries. Of note, the face is the most susceptible area to trauma by road traffic accidents, being the most exposed area of the body. In the study of Aldawsari et al. approximately $60 \%$ of the cohort who sustained maxillofacial trauma suffered from concomitant brain damage [25].

\section{Initial assessment}

TBI could be associated with loss of cognitive, physical, or psychosocial functions leading to decreased level of consciousness, memory impairment, neurologic deficits, and alteration in the mental state of the individual [26]. Most of the MVCs are considered emergency cases and the first and foremost protocol in management is the maintenance of airway, breathing, circulation, stabilization of the disability, and prevention of secondary brain injury. In this situation, the initial assessment includes resuscitation and neurological examination [27].

\section{Prognosis}

The management of severe TBI is complex and overwhelming. Although several prognostic models have been developed and tested, the accurate assessment of short and long-term prognosis remains poorly validated. There is a need to properly understand the molecular mechanisms of TBI, which would help healthcare providers to accurately predict the outcomes [28].

\section{Limitations}

Our study was retrospective and hospital-based, hence could have some limitations of its own. There is a chance of selection bias and misclassification that could arise while retrospectively processing the data. Second, the type of management the patient received during hospitalization was not detailed, which might influence recovery. Despite these limitations, the present study highlighted that motor vehicle accidents and falls were the leading causes of TBI, thus emphasizing the importance of considering the implementation of preventive measures and appropriate public health programs including traffic safety rules. This helps to develop educational programs for neurotrauma prevention to be utilized as a blueprint for local hospitals and officials in Saudi Arabia.

\section{Conclusions}

The leading cause of traumatic brain injury in Saudi Arabia is motor vehicle accidents, followed by falls. The public should be made aware of road traffic rules and safety practices in motor vehicle accidents. People working at heights, such as building construction workers, have a high potential for falls and should be properly instructed to take precautionary and safety measures, i.e., protective equipment, in order to reduce the impact of traumatic brain injury.

\section{Additional Information \\ Disclosures}

Human subjects: Consent was obtained or waived by all participants in this study. Research and Studies Department, Directorate of Health Affairs issued approval HAP-02-T-067. The ethical committees of the Research and Studies Department, Directorate of Health Affairs approved the current study. Prior to the commencement of the study, permission was obtained from King Faisal Medical Complex and King AbdulAziz Specialized Hospital. Patient-related data were preserved and kept confidential. The identifying personal details were not included in the analysis. The assigned protocol number is "HAP-02-T-067". Animal subjects: All authors have confirmed that this study did not involve animal subjects or tissue. 
Conflicts of interest: In compliance with the ICMJE uniform disclosure form, all authors declare the following: Payment/services info: All authors have declared that no financial support was received from any organization for the submitted work. Financial relationships: All authors have declared that they have no financial relationships at present or within the previous three years with any organizations that might have an interest in the submitted work. Other relationships: All authors have declared that there are no other relationships or activities that could appear to have influenced the submitted work.

\section{Acknowledgements}

The authors would like to express their gratitude to Rakan Faisel Almnjwami, Rashed Ayed Althobaiti, Ahmad Saeed A Alghamdi, and Mohammed Abdullah Alswat for their continuous assistance in data acquisition throughout the project.

\section{References}

1. Coburn K: Traumatic brain injury: the silent epidemic . AACN Clin Issues Crit Care Nurs. 1992, 3:9-18. 10.4037/15597768-1992-1002

2. Pavlova V, Filipova E, Uzunova K, Kalinov K, Vekov T: Pioglitazone therapy and fractures: systematic review and meta-analysis. Endocr Metab Immune Disord Drug Targets. 2018, 18:502-7. 10.2174/1871530318666180423121833

3. Dewan MC, Rattani A, Gupta S, et al.: Estimating the global incidence of traumatic brain injury . J Neurosurg. 2018, 1-18. 10.3171/2017.10.JNS17352

4. Popescu C, Anghelescu A, Daia C, Onose G: Actual data on epidemiological evolution and prevention endeavours regarding traumatic brain injury. J Med Life. 2015, 8:272-7.

5. Arabi YM, Haddad S, Tamim HM, et al.: Mortality reduction after implementing a clinical practice guidelines-based management protocol for severe traumatic brain injury. J Crit Care. 2010, 25:190-5. 10.1016/j.jcrc.2009.05.004

6. Al-Naami MY, Arafah MA, Al-Ibrahim FS: Trauma care systems in Saudi Arabia: an agenda for action . Ann Saudi Med. 2010, 30:50-8. 10.4103/0256-4947.59374

7. Al-Kuwaiti A, Hefny AF, Bellou A, Eid HO, Abu-Zidan FM: Epidemiology of head injury in the United Arab Emirates. Ulus Travma Acil Cerrahi Derg. 2012, 18:213-8. 10.5505/tjtes.2012.03710

8. Kraus JF, McArthur DL: Epidemiologic aspects of brain injury. Neurol Clin. 1996, 14:435-50. 10.1016/s07338619(05)70266-8

9. Traumatic Brain Injury in the United States . (2002). Accessed: Nov 1, 2020: https://www.cdc.gov/TraumaticBrainInjury/.

10. Shackford SR, Mackersie RC, Holbrook TL, Davis JW, Hollingsworth-Fridlund P, Hoyt DB, Wolf PL: The epidemiology of traumatic death. A population-based analysis. Arch Surg. 1993, 128:571-5. 10.1001/archsurg.1993.01420170107016

11. Gennarelli TA, Champion HR, Copes WS, Sacco WJ: Comparison of mortality, morbidity, and severity of 59,713 head injured patients with 114,447 patients with extracranial injuries. J Trauma. 1994, 37:962-8. 10.1097/00005373-199412000-00016

12. McMahon CG, Yates DW, Campbell FM, Hollis S, Woodford M: Unexpected contribution of moderate traumatic brain injury to death after major trauma. J Trauma. 1999, 47:891-5. 10.1097/00005373199911000-00013

13. Patel HC, Bouamra O, Woodford M, King AT, Yates DW, Lecky FE: Trends in head injury outcome from 1989 to 2003 and the effect of neurosurgical care: an observational study. Lancet. 2005, 366:1538-44. 10.1016/S0140-6736(05)67626-X

14. McDonald SJ, Sun M, Agoston DV, Shultz SR: The effect of concomitant peripheral injury on traumatic brain injury pathobiology and outcome. J Neuroinflammation. 2016, 13:90. 10.1186/s12974-016-0555-1

15. Watanabe T, Kawai Y, Iwamura A, Maegawa N, Fukushima H, Okuchi K: Outcomes after traumatic brain injury with concomitant severe extracranial injuries. Neurol Med Chir (Tokyo). 2018, 58:393-9. 10.2176/nmc.oa.2018-0116

16. Teasdale G, Jennett B: Assessment of coma and impaired consciousness. A practical scale . Lancet. 1974, 2:81-4. 10.1016/s0140-6736(74)91639-0

17. Meng X, Shi B: Traumatic brain injury patients with a Glasgow coma scale score of $\leqslant 8$, cerebral edema, and/or a basal skull fracture are more susceptible to developing hyponatremia. J Neurosurg Anesthesiol. 2016, 28:21-6. 10.1097/ANA.0000000000000192

18. Sadaka F, Jadhav A, Miller M, Saifo A, O'Brien J, Trottier S: Is it possible to recover from traumatic brain injury and a Glasgow coma scale score of 3 at emergency department presentation?. Am J Emerg Med. 2018, 36:1624-6. 10.1016/j.ajem.2018.01.051

19. Lieberman JD, Pasquale MD, Garcia R, Cipolle MD, Mark Li P, Wasser TE: Use of admission Glasgow coma score, pupil size, and pupil reactivity to determine outcome for trauma patients. J Trauma. 2003, 55:443436. 10.1097/01.TA.0000081882.79587.17

20. Tien HC, Cunha JR, Wu SN, Chughtai T, Tremblay LN, Brenneman FD, Rizoli SB: Do trauma patients with a Glasgow coma scale score of 3 and bilateral fixed and dilated pupils have any chance of survival?. J Trauma. 2006, 60:274-8. 10.1097/01.ta.0000197177.13379.f4

21. Ohwaki K, Yano E, Nagashima H, Nakagomi T, Tamura A: Impact of infection on length of intensive care unit stay after intracerebral hemorrhage. Neurocrit Care. 2008, 8:271-5. 10.1007/s12028-007-9007-1

22. Kleiven S, Peloso PM, von Holst H: The epidemiology of head injuries in Sweden from 1987 to 2000 . Inj Control Saf Promot. 2003, 10:173-80. 10.1076/icsp.10.3.173.14552

23. Al-Habib A, Attabib N, Hurlbert RJ: Recreational helmet use as a predictor of noncranial injury . J Trauma Acute Care Surg. 2012, 72:1356-62. 10.1097/TA.0b013e318250b537

24. El-Matbouly M, El-Menyar A, Al-Thani H, et al.: Traumatic brain injury in Qatar: age matters--insights from 


\section{Cureus}

a 4-year observational study. ScientificWorldJournal. 2013, 2013:354920. 10.1155/2013/354920

25. Aldwsari OM, Aldosari KH, Alzahrani MK, et al.: Associated head injuries and survival rate of patients with maxillofacial fractures in road traffic accident: a prospective study in Saudi Arabia. J Family Med Prim Care. 2018, 7:1548-54. 10.4103/jfmpc.jfmpc_101_18

26. Menon DK, Schwab K, Wright DW, Maas AI: Position statement: definition of traumatic brain injury . Arch Phys Med Rehabil. 2010, 91:1637-40. 10.1016/j.apmr.2010.05.017

27. Pervez M, Kitagawa RS, Chang TR: Definition of traumatic brain injury, neurosurgery, trauma orthopedics, neuroimaging, psychology, and psychiatry in mild traumatic brain injury. Neuroimaging Clin N Am. 2018, 28:1-13. 10.1016/j.nic.2017.09.010

28. El-Menyar A, Mekkodathil A, Al-Thani H, Consunji R, Latifi R: Incidence, demographics, and outcome of traumatic brain injury in the Middle East: a systematic review. World Neurosurg. 2017, 107:6-21.

10.1016/j.wneu.2017.07.070 